\title{
The Einstein-Vlasov system in spherical symmetry: reduction of the equations of motion and classification of single-shell static solutions, in the limit of massless particles
}

\author{
Carsten Gundlach \\ Mathematical Sciences, University of Southampton, Southampton SO17 1BJ, United Kingdom
}

(Dated: 27 October 2016, revised 7 December 2016)

\begin{abstract}
We express the Einstein-Vlasov system in spherical symmetry in terms of a dimensionless momentum variable $z$ (radial over angular momentum). This regularises the limit of massless particles, and in that limit allows us to obtain a reduced system in independent variables $(t, r, z)$ only. Similarly, in this limit the Vlasov density function $f$ for static solutions depends on a single variable $Q$ (energy over angular momentum). This reduction allows us to show that any given static metric which has vanishing Ricci scalar, is vacuum at the centre and for $r>3 M$ and obeys certain energy conditions uniquely determines a consistent $f=\bar{k}(Q)$ (in closed form). Vice versa, any $\bar{k}(Q)$ within a certain class uniquely determines a static metric (as the solution of a system of two first-order quasilinear ODEs). Hence the space of static spherically symmetric solutions of Einstein-Vlasov is locally a space of functions of one variable. For a simple 2-parameter family of functions $\bar{k}(Q)$, we construct the corresponding static spherically symmetric solutions, finding that their compactness is in the interval $0.7 \lesssim \max _{\mathrm{r}}(2 \mathrm{M} / \mathrm{r}) \leq 8 / 9$. This class of static solutions includes one that agrees with the approximately universal type-I critical solution recently found by Akbarian and Choptuik (AC) in numerical time evolutions. We speculate on what singles it out as the critical solution found by fine-tuning generic data to the collapse threshold, given that $\mathrm{AC}$ also found that all static solutions are one-parameter unstable and sit on the threshold of collapse.
\end{abstract}

\section{CONTENTS}

I. Introduction

II. The Einstein-Vlasov system in spherical symmetry
A. Field equations
B. New variables
C. Static solutions

III. The massless case
A. The reduced system
B. Static solutions
C. From metric to matter: Volterra equations
D. From matter to metric: ODE boundary value problems

IV. Examples of static massless solutions
A. The thin-shell solution
B. Ansatz 1: $\varphi(q)$ a simple power
C. Numerical examples of Ansatz 1

V. The space of static solutions

VI. The critical solution

VII. Conclusions

Acknowledgments

A. Dimensional analysis

B. Expansion of the shooting equations about $u_{1}$

C. Ansatz 2: Akbarian and Choptuik
D. Ansatz 3: Andréasson, Fajman and Thaller

\section{INTRODUCTION}

This work is an attempt to better understand some interesting recent numerical results by Akbarian and Choptuik [1] (from now on, AC) on type-I critical collapse in the spherically Einstein-Vlasov system with massless particles. (For a general review of critical collapse, see [2. Critical collapse in the same system with massive particles was considered in [3.) We begin with a brief summary of what we consider their two key results.

(I) Evolving several families of initial data for the massless spherically symmetric Einstein-Vlasov system, AC found that they could fine-tune any one parameter of these initial data sets to the collapse threshold. They found type-I critical collapse with a static critical solution that has an approximately universal metric after rescaling to $M=1$, but not a universal matter distribution $f$. This critical metric has compactness $\Gamma \simeq 0.80 \pm 0.01$ and central redshift $Z_{c} \simeq 2.45 \pm 0.05$. The lifetime scaling exponent is also approximately universal with value $\sigma \simeq 1.4 \pm 0.1$. It is not clear if the small variations of the critical spacetime and critical exponents are numerical error or a genuine variation.

(II) In a separate series of numerical experiments, AC constructed static solutions by specifying the matter distribution and solving for the metric. The resulting metrics are clearly not universal, with compactness and central redshift in the ranges $0.80 \lesssim \Gamma \lesssim 0.89$ and $2.0 \lesssim Z_{c} \lesssim 2.4$. AC then evolved these static solu- 
tions plus a small perturbation from one of three families. They found that all their static solutions are at the threshold of collapse, in the sense that for one sign of the perturbation they collapse and for the other sign they disperse. They also found lifetime scaling with $\sigma \simeq 1.43 \pm 0.07$, consistent with the existence of a single unstable mode with a universal value of its Lyapunov exponent equal to the one seen in fine-tuned generic initial data.

In this paper we clarify the nature of the space of static spherically symmetric massless solutions, leaving their perturbations to future work.

In Sec. II we cast the spherical Einstein-Vlasov equation in a new form, based on an idea in [5]. (In the meantime, a similar formulation has been given in 66.) The key idea is to introduce independent variables $(t, r, z, F)$ such that the particle mass $m$ and particle angular momentum squared $F$ appear only in the combination $m^{2} / F$. The purpose of this section is to establish notation and to demonstrate that the new formalism is as natural as the standard one for $m>0$. In Sec. III, we take the limit $m=0$, in which $F$ can be integrated out. We then restrict this reduced system to static solutions and show that given one of the following three functions - a static density function $f=\bar{k}(Q)$, or one of the two metric coefficients $a_{0}(r), \alpha_{0}(r)$ of a generic static spherically symmetric metric - one can uniquely obtain the other two.

In Sec. IV we numerically construct examples of static massless solutions for a simple 2-parameter family of functions $\bar{k}(Q)$. In Sec. V we formalise our theoretical and numerical insights into three conjectures about the space of static solutions. One particular static solution we have constructed agrees well with the (approximately) universal critical solution found by AC in their time evolutions, to within their numerical accuracy. In Sec. VI we speculate on what singles out this critical solution actually a class of solutions with the same leading order behaviour — from all other static solutions.

In Sec. VII we summarise, and discuss the relation of our results to those of $\mathrm{AC}$.

Appendixes $\mathrm{A}$ and $\mathrm{B}$ contain technical details that would disrupt the main argument, and Appendixes $\mathrm{C}$ and $\mathrm{D}$ express other ansätze for static solutions from the literature in our notation. Throughout this paper, $x:=y$ means that $x$ is being defined, and the suffix 0 denotes static solutions.

\section{THE EINSTEIN-VLASOV SYSTEM IN SPHERICAL SYMMETRY}

\section{A. Field equations}

The Einstein-Vlasov system describes the evolution of a statistical ensemble of non-interacting particles coupled to gravity through their average stress-energy. We restrict ourselves to a single species of particles with mass $m \geq 0$.

We describe the state of a many-body system with a distribution function over the phase space of the system. As the particles are equivalent and uncorrelated, we can use the one-particle distribution function $f\left(x^{\mu}, p_{\nu}\right)$, where $x^{\mu}$ are coordinates in spacetime and $p_{\mu}$ are coordinates in the cotangent space at that point. Because of the constraint $p^{\mu} p_{\mu}=-m^{2}$, we can consider the distribution $f$ as a function of $\left(x^{\mu}, p^{i}\right)$.

Each particle follows a geodesic. This defines a congruence of curves on phase space, tangent to the Liouville operator (or "geodesic spray")

$$
\begin{aligned}
\frac{d}{d \sigma} & :=\frac{d x^{\mu}}{d \sigma} \frac{\partial}{\partial x^{\mu}}+\frac{d p^{i}}{d \sigma} \frac{\partial}{\partial p^{i}} \\
& =p^{\mu} \frac{\partial}{\partial x^{\mu}}-\Gamma_{\nu \lambda}^{i} p^{\nu} p^{\lambda} \frac{\partial}{\partial p^{i}}
\end{aligned}
$$

Here $\sigma$ is an affine parameter related to proper time $s$ through $d s=m d \sigma$ for massive particles. This allows us to treat massless particles as the limit $m \rightarrow 0$ of massive ones. As the particles do not interact directly, the evolution of $f$ is governed by the Vlasov equation

$$
\mathcal{L} f=0 .
$$

The Einstein equations are $G_{\mu \nu}=8 \pi T_{\mu \nu}$, with the stress-energy tensor of collisionless matter given by

$$
T_{\mu \nu}=\int p_{\mu} p_{\nu} f d V_{p}
$$

The invariant volume element on the mass shell in 4momentum space can be written as

$$
d V_{p}=2 \sqrt{-g} \delta\left(p_{\mu} p^{\mu}+m^{2}\right) d^{4} p^{\mu}=\frac{\sqrt{-g} d^{3} p^{i}}{p_{t}} .
$$

The Vlasov equation is a sufficient condition for stressenergy conservation. The particle number current given by

$$
N^{\mu}=\int p^{\mu} f d V_{p}
$$

is also conserved.

Now we impose spherical symmetry. We write the generic spherically symmetric metric in polar-radial coordinates, where it takes the form

$$
d s^{2}=-\alpha^{2}(t, r) d t^{2}+a^{2}(t, r) d r^{2}+r^{2}\left(d \theta^{2}+\sin ^{2} \theta d \varphi^{2}\right) .
$$

In this coordinate choice there is a residual gauge freedom $t \rightarrow t^{\prime}(t)$, which changes the lapse $\alpha$. We fix this freedom by setting $\alpha(t, \infty)=1$. The Einstein equations give the following equations for the first derivatives of the metric coefficients:

$$
\begin{aligned}
\frac{\alpha_{, r}}{\alpha} & =\frac{a^{2}-1}{2 r}+4 \pi r a^{2} T_{r}^{r}, \\
\frac{a_{, r}}{a} & =-\frac{a^{2}-1}{2 r}-4 \pi r a^{2} T_{t}{ }^{t}, \\
\frac{a_{, t}}{a} & =4 \pi r a^{2} T_{t}^{r} .
\end{aligned}
$$


The fourth Einstein equation, involving $T_{\theta}{ }^{\theta}$, is a combination of derivatives of these three, and so is redundant.

As geometric observables, we define the Hawking mass

$$
M_{H}(t, r):=\frac{r}{2}\left[1-a^{-2}(t, r)\right]
$$

and, for static solutions $\left(a_{0}, \alpha_{0}\right)$, following the notation in the literature, the maximum compactness

$$
\Gamma:=\max _{r} \frac{2 M_{H}(r)}{r}=1-\left[\max _{r} a_{0}(r)\right]^{-2}
$$

and the central lapse and, equivalently, central redshift

$$
\alpha_{c}:=\alpha_{0}(0), \quad Z_{c}:=\frac{1}{\alpha_{c}}-1
$$

(not to be confused with the dynamical variable $Z$ introduced below).

In spherical symmetry, the distribution function has to be of the form $f\left(t, r, p_{r},|p|\right)$, where $|p|^{2}:=g_{i j} p^{i} p^{j}$. As is well-known, we can simplify the Vlasov equation using the fact that angular momentum is a constant of motion. We denote its square by

$$
F:=r^{2}\left|p_{\|}\right|^{2}=r^{4}\left[\left(p^{\theta}\right)^{2}+\sin ^{2} \theta\left(p^{\varphi}\right)^{2}\right],
$$

where $p_{\|}$is the 3 -momentum vector tangential to the symmetry orbits of constant $r$. The Vlasov equation and stress-energy tensor in terms of $f\left(t, r, p_{r}, F\right)$ are given, for example, in [5], and we do not repeat them here. Instead we adopt a new set of variables designed to make the limit of vanishing particle mass $m$ transparent and to simplify the equations in that limit.

\section{B. New variables}

To motivate the new variables, we note that the massless Einstein-Vlasov system has an additional symmetry. Two massless point particles with the same starting point and with initial 4-momenta $p_{\mu}$ and $\lambda p_{\mu}$, for any $\lambda>0$, follow the same worldline. As a consequence, if two Vlasov distributions $f$ and $f^{\prime}$ are related by $f^{\prime}\left(x^{\mu}, p_{\mu}\right)=\lambda^{4} f\left(x^{\mu}, \lambda p_{\mu}\right)$ we see from (4) that they give rise to the same stress-energy tensor $T_{\mu \nu}$, and so are consistent with the same metric. (This invariance does not hold for massive particles because the rest mass $m$ does not scale. Put differently, two massive particles with initial 4-momenta $p_{\mu}$ and $\lambda p_{\mu}$ follow different trajectories.)

To exploit this scale-invariance of the massless case in practice, we need to be able to integrate out a particle momentum component that is also a constant of the motion. In spherical symmetry, the absolute value $\sqrt{F}$ of the particle angular momentum is conserved along particle trajectories, and so can play this role, even in the time-dependent case. Hence we replace the radial momentum $p_{r}$ by

$$
z:=\frac{p_{r}}{a \sqrt{F}}
$$

(The factor $a$ has been introduced for convenience.) It is easy to see that for massless particles this is invariant under rescaling $p_{\mu}$. The Vlasov equation for $f(t, r, z, F)$ is then

$$
\frac{\partial f}{\partial t}+\frac{\alpha z}{a Z} \frac{\partial f}{\partial r}+\left(\frac{\alpha}{r^{3} a Z}-\frac{\alpha_{, r} Z}{a}-\frac{z a_{, t}}{a}\right) \frac{\partial f}{\partial z}=0
$$

where we have defined the shorthand

$$
Z(r, z, F):=\sqrt{\frac{m^{2}}{F}+z^{2}+\frac{1}{r^{2}}} .
$$

The partial derivative $\partial / \partial F$ does not appear in the Vlasov equation because $F$ is a constant of motion. Furthermore, $m$ and $F$ appear in the Vlasov equation only in the combination $m^{2} / F$. Hence the limit $m=0$ is regular.

Parameterising the tangential momentum in terms of $F$ and the angle $0 \leq \chi<2 \pi$ in the plane of tangential momenta,

$$
p^{\theta}=: \frac{\sqrt{F}}{r^{2}} \cos \chi, \quad \sin \theta p^{\varphi}=: \frac{\sqrt{F}}{r^{2}} \sin \chi,
$$

we have

$$
d p^{\theta} \wedge d\left(\sin \theta d p^{\varphi}\right)=\frac{d F \wedge d \chi}{2 r^{4}} .
$$

With this intermediate step, it is then easy to see that the momentum space volume element $d V_{p}$ given in (5) in can be written in the new variables as

$$
d V_{p}=\frac{d F \wedge d z \wedge d \chi}{2 r^{2} Z} .
$$

With

$$
\begin{aligned}
\int_{\chi=0}^{2 \pi} d V_{p} & =\frac{\pi d F \wedge d z}{r^{2} Z}, \\
\int_{\chi=0}^{2 \pi} \sin ^{2} \chi d V_{p} & =\frac{\pi d F \wedge d z}{2 r^{2} Z},
\end{aligned}
$$

the non-vanishing components of the stress-energy tensor are given by the integrals

$$
\begin{aligned}
p & :=T_{r}{ }^{r}=\frac{\pi}{r^{2}} \int_{0}^{\infty} F d F \int_{-\infty}^{\infty} f \frac{z^{2}}{Z} d z \geq 0(23) \\
\rho:=-T_{t}{ }^{t} & =\frac{\pi}{r^{2}} \int_{0}^{\infty} F d F \int_{-\infty}^{\infty} f Z d z \geq 0,(24) \\
j & :=T_{t}{ }^{r}=-\frac{\pi \alpha}{a r^{2}} \int_{0}^{\infty} F d F \int_{-\infty}^{\infty} f z d z, \quad(25) \\
p_{T}:=T_{\theta}{ }^{\theta} & =T_{\varphi}{ }^{\varphi}=\frac{\pi}{2 r^{4}} \int_{0}^{\infty} F d F \int_{-\infty}^{\infty} f \frac{1}{Z} d z \geq 0(26)
\end{aligned}
$$

We note that in the Einstein equations $m$ and $F$ appear only in the combinations $m^{2} / F$ and $F d F$. Again the limit $m=0$ is regular.

$\rho, p, p_{T}$ and $j$ are the energy density, radial and tangential pressure and energy current observed by observers at constant $r$. We assume that $f \geq 0$ and that $f$ behaves 
in such a way that these integrals exist and are finite at every point in spacetime. $T_{\mu}{ }^{\nu}$ is conserved and satisfies the dominant and strong energy conditions. It is easy to see that $T_{\mu}{ }^{\mu}=0$, or $\rho+p+2 p_{T}=0$, if $m=0$. The Einstein equations 910 can be rewritten in terms of $M_{H}$, $\rho$ and $j$ in the pseudo-Newtonian form

$$
\begin{aligned}
M_{H, r} & =4 \pi r^{2} \rho, \\
M_{H, t} & =4 \pi r^{2} j .
\end{aligned}
$$

\section{Static solutions}

A static spacetime has an additional Killing vector

$$
\xi:=\frac{\partial}{\partial t}
$$

and hence in our coordinates the metric takes the form

$$
d s^{2}=-\alpha_{0}^{2}(r) d t^{2}+a_{0}^{2}(r) d r^{2}+r^{2}\left(d \theta^{2}+\sin ^{2} \theta d \varphi^{2}\right) .
$$

The Einstein equations are then

$$
\begin{aligned}
& \frac{\alpha_{0}^{\prime}}{\alpha_{0}}=\frac{a_{0}^{2}-1}{2 r}+4 \pi r a^{2} p_{0}, \\
& \frac{a_{0}^{\prime}}{a_{0}}=-\frac{a_{0}^{2}-1}{2 r}+4 \pi r a^{2} \rho_{0} .
\end{aligned}
$$

In a static spacetime, the particles have an additional constant of motion

$$
E:=-\xi^{\mu} p_{\mu}=-p_{t}=\alpha_{0} \sqrt{F} Z .
$$

A self-consistent static solution can be obtained from the ansatz

$$
f\left(r, p_{r}, F\right)=h(E, F) .
$$

Static solutions with this ansatz for $m>0$, including solutions with matter at the centre of spherical symmetry ("non-shells") and with multiple (thick) shells separated by vacuum regions have been constructed in 4 .

In Newtonian physics, this ansatz includes all possible static solutions, a result known as Jean's theorem, but in GR the most general self-consistent static solution of Einstein-Vlasov in spherical symmetry has the form [5]

$$
f\left(r, p_{r}, F\right)=\sum_{n} \theta\left(r-r_{-}^{(n)}\right) \theta\left(r_{+}^{(n)}-r\right) h^{(n)}(E, F),
$$

where the limits $r_{ \pm}^{(n)}$ of the $n$-th (thick) shell depend on $h^{(n)}$ and the total mass further inside. In other words, in GR there can be more than one potential well, labelled by $(n)$, each with a different Vlasov distribution function $h^{(n)}(E, F)$. For simplicity of notation only, in the following we focus on the case of a single potential well. Multiwell solutions can be constructed by surrounding an existing solution with an additional matter shell further out, taking into account that just inside the additional shell the spacetime is not Minkowski but Schwarzschild.
Instead of using $E$, we write the Vlasov function of a static spherically symmetric solution as

$$
f(r, z, F)=k(Q, F),
$$

where

$$
Q(r, z, F):=\frac{E^{2}}{F}=\alpha_{0}^{2} Z^{2}
$$

plays a similar role to $E$ but is invariant under rescaling $p_{\mu}$ for massless particles. We also change the integration variables in the stress-energy from $z$ and $F$ to $Q$ and $F$. This gives the stress-energy components

$$
\begin{aligned}
& p_{0}=\frac{\pi}{\alpha_{0}^{2} r^{2}} \int_{0}^{\infty} F d F \int_{U}^{\infty} k v d Q, \\
& \rho_{0}=-\frac{\pi}{\alpha_{0}^{2} r^{2}} \int_{0}^{\infty} F d F \int_{U}^{\infty} k \frac{1}{v} d Q,
\end{aligned}
$$

where we have defined the shorthands

$$
\begin{aligned}
U(r, F) & :=\alpha_{0}^{2}\left(\frac{m^{2}}{F}+\frac{1}{r^{2}}\right), \\
v(r, Q, F) & :=\frac{z}{Z}=\sqrt{1-\frac{U}{Q}}=\frac{\frac{\left|p_{r}\right|}{a_{0}}}{\sqrt{\frac{p_{r}^{2}}{a_{0}^{2}}+\frac{F}{r^{2}}}}
\end{aligned}
$$

for the integration limit and integral kernel. Physically, $U$ is an effective potential for the radial particle motion in the sense that

$$
z^{2}=\alpha_{0}^{-2}(Q-U)
$$

and hence $Q=U$ determines the radial turning point for all particles with a given $Q$ and $F$. From the last equality in 41 we see that physically $\pm v$ is the radial particle speed, expressed in units of the speed of light and measured by observers at constant $r$.

\section{THE MASSLESS CASE}

\section{A. The reduced system}

We now restrict to the case $m=0$. The key observation is that $Z$ then becomes independent of $F$. Hence $F$ no longer appears in the Vlasov equation (16) at all. This has two key consequences: first, the integrated Vlasov density

$$
\bar{f}(t, r, z):=\int_{0}^{\infty} f(t, r, z, F) F d F
$$

obeys the same PDE as $f$ itself, namely

$$
\frac{\partial \bar{f}}{\partial t}+\frac{\alpha z}{a Z} \frac{\partial \bar{f}}{\partial r}+\left(\frac{\alpha}{r^{3} a Z}-\frac{\alpha_{, r} Z}{a}-\frac{z a, t}{a}\right) \frac{\partial \bar{f}}{\partial z}=0
$$

where now

$$
Z(r, z)=\sqrt{z^{2}+\frac{1}{r^{2}}}
$$


Secondly, the integration limit $U$ and kernel $v$ in the double integrals for the stress-energy tensor become independent of $F$, and we can therefore change the order of integration and write

$$
\begin{aligned}
p & =\frac{\pi}{r^{2}} \int_{-\infty}^{\infty} \bar{f} \frac{z^{2}}{Z} d z \geq 0, \\
\rho & =-\frac{\pi}{r^{2}} \int_{-\infty}^{\infty} \bar{f} Z d z \leq 0, \\
j & =-\frac{\pi \alpha}{a r^{2}} \int_{-\infty}^{\infty} \bar{f} z d z \\
p_{T} & =\frac{\pi}{2 r^{4}} \int_{-\infty}^{\infty} \bar{f} \frac{1}{Z} d z \geq 0 .
\end{aligned}
$$

We now have a closed system of field equations for the unknowns $a(t, r), \alpha(t, r)$ and $\bar{f}(t, r, z)$. We shall call this the reduced system. For any solution $a(t, r), \alpha(t, r)$ and $f(t, r, z, F)$ of the full system, there are infinitely many other solutions $a(t, r), \alpha(t, r)$ and $f^{\prime}(t, r, z, F)$, all corresponding to the same solution of the reduced system, $\bar{f}(t, r, z)=\bar{f}^{\prime}(t, r, z)$.

\section{B. Static solutions}

In the static massless case, we can interchange the momentum integrations in the same way. The static Einstein equations become

$$
\begin{aligned}
& \frac{\alpha_{0}^{\prime}}{\alpha_{0}}=\frac{a_{0}^{2}-1}{2 r}+\frac{4 \pi^{2} a_{0}^{2}}{r \alpha_{0}^{2}} \int_{U}^{\infty} \bar{k} v d Q \\
& \frac{a_{0}^{\prime}}{a_{0}}=-\frac{a_{0}^{2}-1}{2 r}+\frac{4 \pi^{2} a_{0}^{2}}{r \alpha_{0}^{2}} \int_{U}^{\infty} \bar{k} \frac{1}{v} d Q
\end{aligned}
$$

where we have defined

$$
\bar{k}(Q):=\int_{0}^{\infty} k(Q, F) F d F,
$$

and where now

$$
U(r)=\frac{\alpha_{0}^{2}}{r^{2}}
$$

$Q=U(r)$ now gives the turning points of all particles with a given conserved quantity $Q$, independently of $F$.

For simplicity, we again assume that there is only a single potential well in $U$. Throughout the remainder of the paper we will frequently refer to the particular values of $r$ and $U$ illustrated in Fig. 1, and formally defined as follows:

$$
\begin{aligned}
U_{3} & \leq U_{2} \leq U_{1} \leq U_{0} \\
r_{0-} & \leq r_{1-} \leq r_{2-} \leq r_{3} \leq r_{2+} \leq r_{1+} \leq r_{0+} \\
U\left(r_{i \pm}\right) & =U_{i}, \quad i=0,1,2, \quad U\left(r_{3}\right)=U_{3} \\
U^{\prime}\left(r_{3}\right) & =0, \quad U^{\prime \prime}\left(r_{3}\right)>0 \\
U^{\prime}\left(r_{0+}\right) & =0, \quad U^{\prime \prime}\left(r_{0+}\right)<0 \\
\bar{k}(Q) & \neq 0 \quad \Leftrightarrow \quad U_{2}<Q<U_{1} .
\end{aligned}
$$

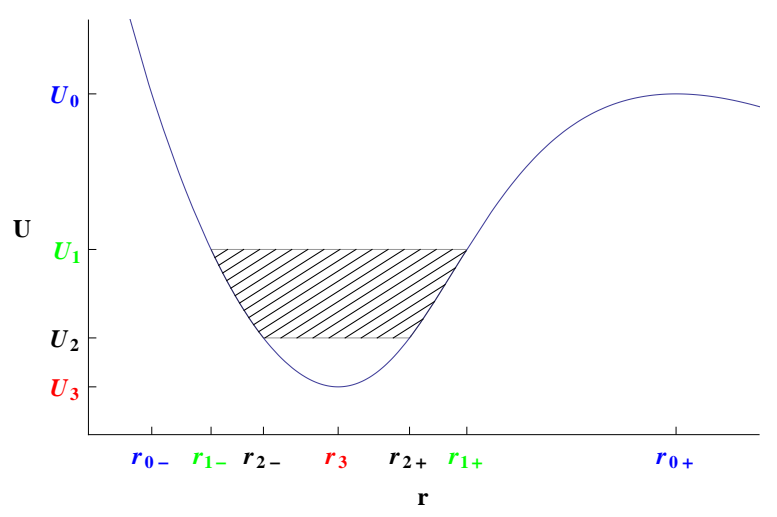

FIG. 1. Sketch of the potential $U(r)$, showing the preferred values of $U$ and $r$ defined in 54.59. The black hatching shows the region in $(U, r)$ space where particles are present.

In words, $U_{0}$ is the top of the effective potential, $U_{3}$ its bottom and $\left[U_{2}, U_{1}\right]$ is the support of $\bar{k}(Q)$, which for bound particles must lie between the top and bottom of the potential. In the limiting case $U_{1}=U_{2}, \bar{k}(Q)$ is a $\delta$-function, while $U_{1}=U_{0}$ and $U_{2}=U_{3}$ correspond to the matter distribution filling the potential well to the lip and to the bottom, respectively.

Fig. 1 also illustrates the following observation. For $U_{1}<U_{0}$, the maximum $r_{0+}$ of the potential is in vacuum, and even for $U_{1}=U_{0}$ it is still in vacuum from one side. Hence it must be identical with the only maximum that the vacuum potential has, also known as the unstable photon orbit of the Schwarzschild solution. From this identification we read off

$$
r_{0+}=3 M, \quad U_{0}=\frac{1}{27 M^{2}} .
$$

For massless particles the Ricci scalar vanishes, and as a consequence $a(t, r)$ and $\alpha(t, r)$ are related by an Einstein equation in which $f$ does not appear. For static solutions, this reduces to the ordinary differential equation (ODE)

$$
\frac{\alpha_{0}^{\prime \prime}}{\alpha_{0}}+\frac{2}{r}\left(\frac{\alpha_{0}^{\prime}}{\alpha_{0}}-\frac{a_{0}^{\prime}}{a_{0}}\right)-\frac{\alpha_{0}^{\prime}}{\alpha_{0}} \frac{a_{0}^{\prime}}{a_{0}}+\frac{1-a_{0}^{2}}{r^{2}}=0 .
$$

Replacing $a_{0}$ by the new dependent variable

$$
b(r):=\frac{1}{a_{0}^{2}}
$$

we see that (61) is linear, inhomogeneous and first order when considered as an ODE for $b$, namely

$$
\left(r \alpha_{0}+\frac{r^{2}}{2} \alpha_{0}^{\prime}\right) b^{\prime}+\left(r^{2} \alpha_{0}^{\prime \prime}+2 r \alpha_{0}^{\prime}+\alpha_{0}\right) b=\alpha_{0}
$$

Given $\alpha_{0}$ and the regularity boundary condition $b(0)=1$, this has a unique solution $b$, which moreover can be given in closed form as an integral. At the same time, (61) is 
linear, homogeneous and second order when considered as an ODE for $\alpha_{0}$, namely

$$
\alpha_{0}^{\prime \prime}+\left(\frac{2}{r}+\frac{b^{\prime}}{2 b}\right) \alpha_{0}^{\prime}+\left(\frac{b^{\prime}}{r b}+\frac{b-1}{r^{2}}\right) \alpha_{0}=0 .
$$

Given $b$, the regularity boundary condition $\alpha_{0}^{\prime}(0)=0$ and the gauge boundary condition $\alpha_{0}(\infty)=1$, this has a unique solution $\alpha_{0}$. For any trace-free matter, and in particular for massless Einstein-Vlasov, $a_{0}(r)$ and $\alpha_{0}(r)$ are therefore related one-to one.

As discussed before, we always normalise $t$ so that it is proper time at infinity. Then in the interior vacuum region $\left[0, r_{1-}\right)$,

$$
\alpha_{0}(r)=\alpha_{\mathrm{c}} \leq 1, \quad a_{0}(r)=1,
$$

while in the exterior vacuum region $\left(r_{1+}, \infty\right)$,

$$
\alpha_{0}(r)=\left(1-\frac{2 M}{r}\right)^{\frac{1}{2}}, \quad a_{0}(r)=\left(1-\frac{2 M}{r}\right)^{-\frac{1}{2}}
$$

where $M$ is the total (ADM) mass.

\section{From metric to matter: Volterra equations}

We define the combination of metric derivatives

$$
\begin{aligned}
& A(r):=\left(\frac{\alpha_{0}^{\prime}}{\alpha_{0}}-\frac{a_{0}^{2}-1}{2 r}\right) \frac{\alpha_{0} r^{2}}{4 \pi^{2} a_{0}^{2}}, \\
& B(r):=\left(\frac{a_{0}^{\prime}}{a_{0}}+\frac{a_{0}^{2}-1}{2 r}\right) \frac{\alpha_{0}^{3}}{4 \pi^{2} a_{0}^{2}},
\end{aligned}
$$

which are related to the stress-energy as

$$
\begin{aligned}
\rho_{0} & :=\frac{\pi}{r \alpha_{0}^{3}} B, \\
p_{0} & :=\frac{\pi}{r^{3} \alpha_{0}} A .
\end{aligned}
$$

In terms of $A$ and $B$, the Einstein equations 50 51) can be written as

$$
\begin{aligned}
& A(r)=\int_{0}^{u(r)} \sqrt{u(r)-q} \varphi(q) d q, \\
& B(r)=\int_{0}^{u(r)} \frac{\varphi(q)}{\sqrt{u(r)-q}} d q,
\end{aligned}
$$

where we have defined the new dependent and independent variables

$$
\varphi(q):=Q^{2} \bar{k}(Q), \quad q:=\frac{1}{Q},
$$

and the shorthand

$$
u(r):=\frac{1}{U}=\frac{r^{2}}{\alpha_{0}^{2}} .
$$

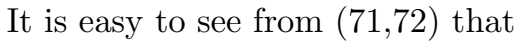

$$
B(r)=2 \frac{A^{\prime}(r)}{u^{\prime}(r)}
$$

and that via 6768 this is equivalent to 61 .

The key observation is now that, from 7172 , for any two values of $r$ where $u(r)$ takes the same value, $A(r)$ must also take the same value, and similarly for $B(r)$. Note this does not hold in the massive case. On each of the two intervals $\left(r_{0-}, r_{3}\right)$ and $\left(r_{3}, r_{0+}\right), u(r)$ is monotonic. Using either one of these intervals, we define the functions $\tilde{A}(u)$ and $\tilde{B}(u)$ on the interval $\left(u_{0}, u_{3}\right)$ by

$$
\tilde{A}[u(r)]:=A(r), \quad \tilde{B}[u(r)]:=B(r) .
$$

We have now turned 50 51 into linear Volterra equations of the first kind, namely

$$
\begin{aligned}
& \tilde{A}(u)=\int_{0}^{u} \sqrt{u-q} \varphi(q) d q, \\
& \tilde{B}(u)=\int_{0}^{u} \frac{\varphi(q)}{\sqrt{u-q}} d q,
\end{aligned}
$$

From 77 they obey

$$
\tilde{B}(u)=2 \tilde{A}^{\prime}(u) .
$$

The integral equation $(78)$ is the Abel equation, which has the unique solution [7, 8

$$
\varphi(q)=\frac{1}{\pi} \frac{d}{d q} \int_{0}^{q} \frac{\tilde{B}(u)}{\sqrt{q-u}} d u .
$$

The unique solution of the integral equation 77 is 8

$$
\varphi(q)=\frac{2}{\pi} \frac{d^{2}}{d q^{2}} \int_{0}^{q} \frac{\tilde{A}(u)}{\sqrt{q-u}} d u .
$$

These two expressions for $\varphi(q)$ are equivalent modulo 79 .

In summary, we can uniquely obtain the matter from "half the metric" in closed form as follows. With either $\alpha_{0}(r)$ or $a_{0}(r)$ given, we solve (61) for the other one. We obtain $A(r)$ and $B(r)$ by differentiation, and hence implicitly $\tilde{A}(u)$ and $\tilde{B}(u)$. Finally, we obtain $\varphi(q)$ from either $\tilde{A}(u)$ or $\tilde{B}(u)$ by integration and differentiation.

\section{From matter to metric: ODE boundary value problems}

To obtain the metric from the matter, we first construct $\tilde{A}(u)$ and $\tilde{B}(u)$ by integration of $\varphi(q)$. We then consider 67 68) with the identifications (76) as an ODE system for $a_{0}(r)$ and $\alpha_{0}(r)$. For convenience, we change the dependent variables from $\alpha_{0}$ to $u$ (because $u$ is the argument of $\tilde{A}$ and $\tilde{B}$ ), and from $a_{0}$ to $b$ (because the 
equations then become linear in $b$ ). With these changes, (67 68) become

$$
\begin{aligned}
r b u^{\prime}+(1-3 b) u+8 \pi^{2} r^{-2} u^{\frac{3}{2}} \tilde{A}(u) & =0, \\
r b^{\prime}-1+b+8 \pi^{2} r^{-2} u^{\frac{3}{2}} \tilde{B}(u) & =0 .
\end{aligned}
$$

We now pose a boundary-value problem on the nonvacuum interval $\left[r_{1-}, r_{1+}\right]$, namely the ODE system 8283 with boundary conditions that arise from matching to Minkowski, (65), in the interior and Schwarzschild, (66), in the exterior. These boundary conditions are

$$
\begin{aligned}
& u\left(r_{1-}\right)=u_{1}, \\
& b\left(r_{1-}\right)=1, \\
& u\left(r_{1+}\right)=u_{1}, \\
& b\left(r_{1+}\right)=1-\frac{2 M}{r_{1+}},
\end{aligned}
$$

where $r_{1-}$ is given by

$$
r_{1-}=\alpha_{c} \sqrt{u_{1}},
$$

and $r_{1+}$ is the unique positive real root of the cubic equation

$$
r_{1+}^{3}-u_{1} r_{1+}+2 M u_{1}=0 .
$$

In the limit $u_{1}=u_{0}=27 M^{2}$, we have $r_{1+}=r_{0+}=3 M$ and $b\left(r_{1+}\right)=1 / 3$.

For given $\varphi(q)$, we now have a boundary value problem of two first-order ODEs with two free parameters $M$ and $\alpha_{c}$. (Recall that the value of $u_{1}$ is a property of the given function $\varphi(q)$, namely, the upper limit of its support). Rather than using $M$ as a parameter, however, we take advantage of the scale-invariance of the massless Einstein-Vlasov equations (see Appendix A to fix $M=1$. We then use $\alpha_{c}$ and an overall constant factor $C$ in $\varphi(q)$ as the free parameters to be determined in the boundary value problem.

\section{EXAMPLES OF STATIC MASSLESS SOLUTIONS}

\section{A. The thin-shell solution}

We begin with the limiting case where all the matter forms a thin shell at the bottom of the potential well. In this limit, $b$ becomes discontinuous at the shell because the mass jumps. By contrast, $u$ must be continuous because the first Israel junction condition - the intrinsic metric is continuous across the shell - is equivalent to continuity of $r$ and $\alpha_{0}$. From the Einstein equations, we then see that $u^{\prime}$ is discontinuous.

In order to derive the thin-shell case as the formal limit of a family of thick shells, we make the ansatz

$$
\begin{aligned}
b(r) & =\hat{b}(x), \\
u(r) & =u_{*}+\epsilon \hat{u}(x), \quad \hat{u}(0)=0
\end{aligned}
$$

where

$$
x:=\frac{r-r_{*}}{\epsilon},
$$

which characterises a shell of width $\epsilon$ centered at $r=r_{*}$. In the limit $\epsilon \rightarrow 0, b(r)$ jumps from $\hat{b}(-\infty)$ to $\hat{b}(\infty)$ $u(r)$ is continuous with value $u_{*}$, and $u^{\prime}(r)$ jumps from $\hat{u}^{\prime}(-\infty)$ to $\hat{u}^{\prime}(\infty)$.

Taking the combination $2 \cdot(82)^{\prime}-u^{\prime} \cdot(83)$ in order to cancel the diverging stress-energy term $\tilde{B}$ with $2 \tilde{A}^{\prime}$, substituting the ansatz 90,92 , and expanding to leading order in $\epsilon$, we obtain that $\hat{b} \hat{u}^{\prime 2}$ is constant in $x$. This means that in the limit $\epsilon \rightarrow 0$ the quantity $b u^{\prime 2}$ is constant in $r$, and hence that it must take the same value on either side of the thin matter shell. Matching $u$ and $b u^{\prime 2}$ from the interior vacuum $(65)$ to the exterior vacuum 66 at some value $r_{*}$, we obtain two algebraic equations that can be solved to find

$$
r_{*}=\frac{9 M}{4}, \quad \alpha_{c}=\frac{1}{3} .
$$

and hence

$$
u_{*}=\frac{729}{16} M^{2} \simeq 45.6 M^{2}, \quad b_{*}=3 .
$$

From $\alpha_{c}$ and $b_{*}$ (which is the global maximum of $b$ ) we have the diagnostic quantities $Z_{c}=2$ and $\Gamma=8 / 9$.

Note that we have not assumed anything about the function $\tilde{A}(u)$, and so the thin shell limit is independent of the family of thick shells from which it is obtained. It has already been shown 9 that the Buchdahl limit for (massless or massive) static Einstein-Vlasov is $\Gamma \leq 8 / 9$ and that the thin-shell solution saturates it.

\section{B. Ansatz 1: $\varphi(q)$ a simple power}

A simple ansatz where we can transform between $\varphi$ and $\tilde{A}$ or $\tilde{B}$ in closed form is

$$
\begin{aligned}
& \varphi(q)=C(k+1) u_{1}^{-1}\left[\frac{q}{u_{1}}-1\right]_{+}^{k}, \\
& \tilde{A}(u)=\tilde{C} u_{1}^{1 / 2}\left[\frac{u}{u_{1}}-1\right]_{+}^{k+\frac{3}{2}}, \\
& \tilde{B}(u)=2 \tilde{A}^{\prime}(u)=\tilde{C}(2 k+3) u_{1}^{-1 / 2}\left[\frac{u}{u_{1}}-1\right]_{+}^{k+\frac{1}{2}}
\end{aligned}
$$

with parameters $u_{1} \geq u_{0}, C$ and $k$. (Note $u_{1}$ has dimension $L^{2}$, while $C$ is dimensionless, see Appendix A.) We have introduced the shorthand notation $[\ldots]_{+}^{k}:=$ $\theta(\ldots)(\ldots)^{k}$ and

$$
\tilde{C}:=\frac{\sqrt{\pi} \Gamma(k+2)}{2 \Gamma\left(k+\frac{5}{2}\right)} C .
$$


In the limit $k \rightarrow-1$ we find

$$
\begin{aligned}
\varphi(q) & =C \delta\left(\frac{q}{u_{1}}-1\right) \\
\tilde{A}(u) & =C u_{1}^{1 / 2}\left[\frac{u}{u_{1}}-1\right]_{+}^{\frac{1}{2}}, \\
\tilde{B}(u) & =C u_{1}^{-1 / 2}\left[\frac{u}{u_{1}}-1\right]_{+}^{-\frac{1}{2}},
\end{aligned}
$$

and in this case $\tilde{C}=C$. [The factor $(k+1)$ has been included in the ansatz 95 to make the limit $C \rightarrow 1$ regular.] We shall call $k=-1$ the "single-orbit" case, as all particles now follow the same trajectory (modulo orbital phase and orientation of the orbital plane). As this single orbit is in general not circular, the cloud of particles still forms a thick shell in physical space $r$. (In the limit $u_{1} \rightarrow u_{*}$ this single orbit becomes circular, and we again obtain the unique thin-shell solution.)

We now consider what range of $k$ is physical. From (69) and (97) we see that for $k>-1 / 2$, the energy density $\rho_{0}$ is continuous with the vacuum value of zero at the vacuum boundaries $r=r_{1 \pm}$, and for $k=-1 / 2$ it is discontinous but finite. Hence for $k \geq-1 / 2$, the system (82 83) is regular at $u_{1}$. For $k<-1 / 2$, we must start off any numerical solution of 82 83 with an expansion about $u=u_{1}$. In Appendix $\mathrm{B}$ we find such approximations for $u_{1}>u_{0}$ and $-1 \leq k<-1 / 2$, but not for $u_{1}=u_{0}$ and $k \leq-1 / 2$. We conclude that the physical range of the parameters $k$ and $u_{1}$ of our ansatz is given by

$$
\begin{cases}k \geq-1, & u_{0}<u_{1}<u_{*}, \\ k>-\frac{1}{2}, & u_{1}=u_{0} .\end{cases}
$$

(We have excluded $u_{1}=u_{*}$ here because we have already treated the thin-shell case separately). From (70) and $(96)$ we then see that, for any $k$, the radial pressure is continuous with the vacuum value of zero and the tangential pressure $p_{T}$ approaches $\rho$. Physically, this is so because particles move with the speed of light, and at the vacuum boundary of a static solution by definition that speed is tangential.

\section{Numerical examples of Ansatz 1}

We now describe numerical solutions of 8283 with $\varphi(q)$ given by (95). To have a good starting guess for the shooting, we have explored the 2-dimensional parameter space along 1-parameter families of solutions, keeping either $u_{1}$ or $k$ fixed.

A side benefit of using $C$ and $\alpha_{c}$ as free parameters is that as long as the Einstein equations are regular at $r_{1 \pm}$, we can shoot from $r_{1+}$ given by 89 to decreasing $r$, and adjust the single free parameter $C$ so that $u=u_{1}$ and $b=b_{1}$ occur at the same $r$, which we identify as $r_{1-} . \alpha_{c}$ is obtained from $r_{1-}$ by 88 . This means that we need

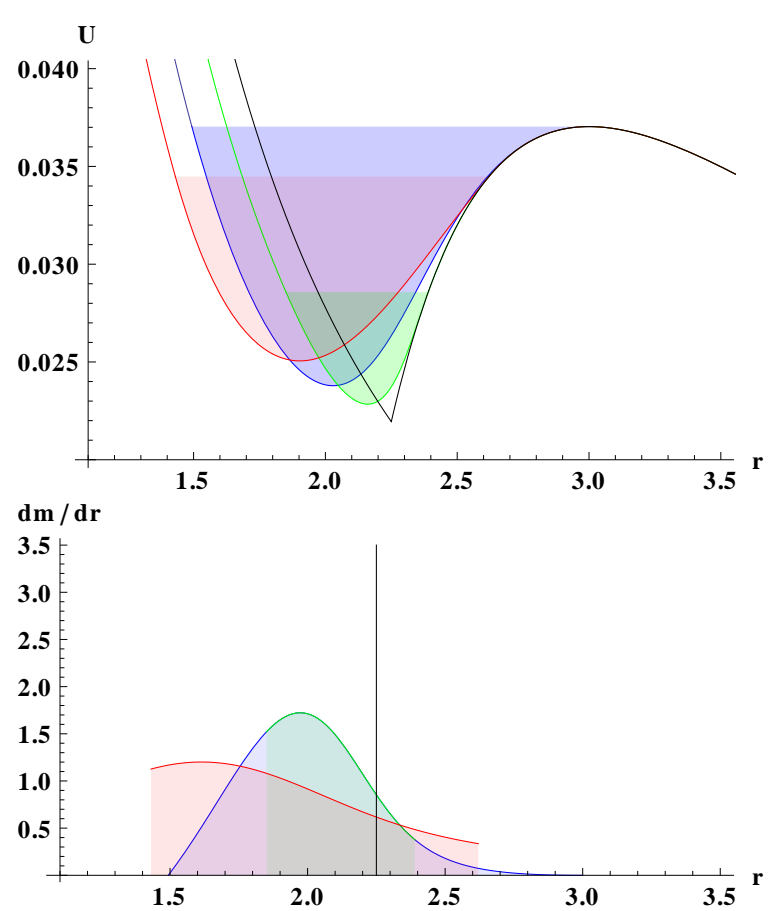

FIG. 2. Effective potential $U(r)$ and mass aspect $d m / d r$ against radius $r$, for four representative solutions (from right to left in the upper plot, and from top to bottom in the lower plot): the thin-shell solution (black, $U^{\prime}(r)$ discontinuous at $r=9 / 4) ;\left(k=1 / 2, u_{1}=35\right)$ (green); $\left(k=1 / 2, u_{1}=27\right)$ (blue, potential well completely filled); and $\left(k=-1 / 2, u_{1}=\right.$ 29) (red). Note that the area under $d m / d r$ is $M=1$ for all four solutions. $d m / d r=\delta(r-9 / 4)$ has been symbolised by a vertical line. Note that the blue solution agrees with the critical solution of AC.

to solve a numerical root-finding problem only in the one parameter $C$.

For $k<-1 / 2$ we start off the integration into the matter region with the approximate solutions for $u \gtrsim u_{1}$ given in Appendix $\mathrm{B}$, and shoot from both $r_{1-}$ and $r_{1+}$ to a midpoint, which we choose as $r=r_{*}$. Our simple implementation of the 2-parameter shooting, using the Mathematica functions NDSolve and FindRoot with their default settings, is not very robust and fails near all three boundaries of the half-strip (102). Hence our coverage of the physical parameter space (102) is incomplete. We improve on this by extrapolating our data points to the entire $k \geq-1, u_{0} \leq u_{1} \leq u_{*}$ half-strip, which for plotting we truncate at $k=4$ (there are no numerical problems at large $k$ ).

Fig. 2 shows the effective potentials $U$ and mass aspect $d m / d r$ for four representative solutions. Fig. 3 shows $r_{1 \pm}$, $r_{0 \pm}$ and $r_{3}$ against $u_{1}$ for $k=1 / 2$. Fig. 4 shows the same locations, but now against both $k$ and $u_{1}$, while Fig. 5 shows $U_{3}$ and $U_{1}$, and Fig. 6 the central lapse $\alpha_{c}$, also against $k$ and $u_{1}$.

From these plots we see that the quantities $\Gamma, \alpha_{c}, r_{0-}$, $r_{1 \pm}$ and $r_{3}$ characterising the shape of the potential well are all monotonic in both $k$ and $u_{1}$. In particular, smaller 


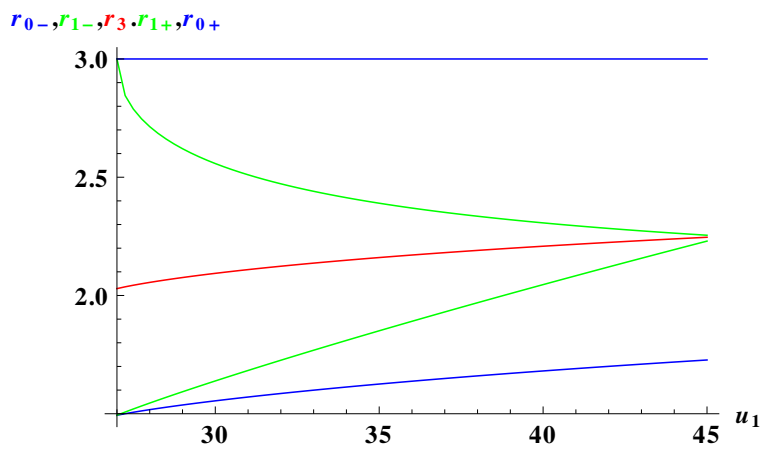

FIG. 3. From bottom to top, $r_{0-}$ (blue), $r_{1-}$ (green), $r_{3}$ (red), $r_{1+}$ (green) and $r_{0+}=3$ (blue) against $u_{1}$ ranging from $u_{0}=$ 27 (the potential well is completely filled) to $u_{*}=729 / 16$ (the thin-shell solution), for Ansatz 1 with $k=1 / 2$. The colour-coding is the same as in Fig. 1 .

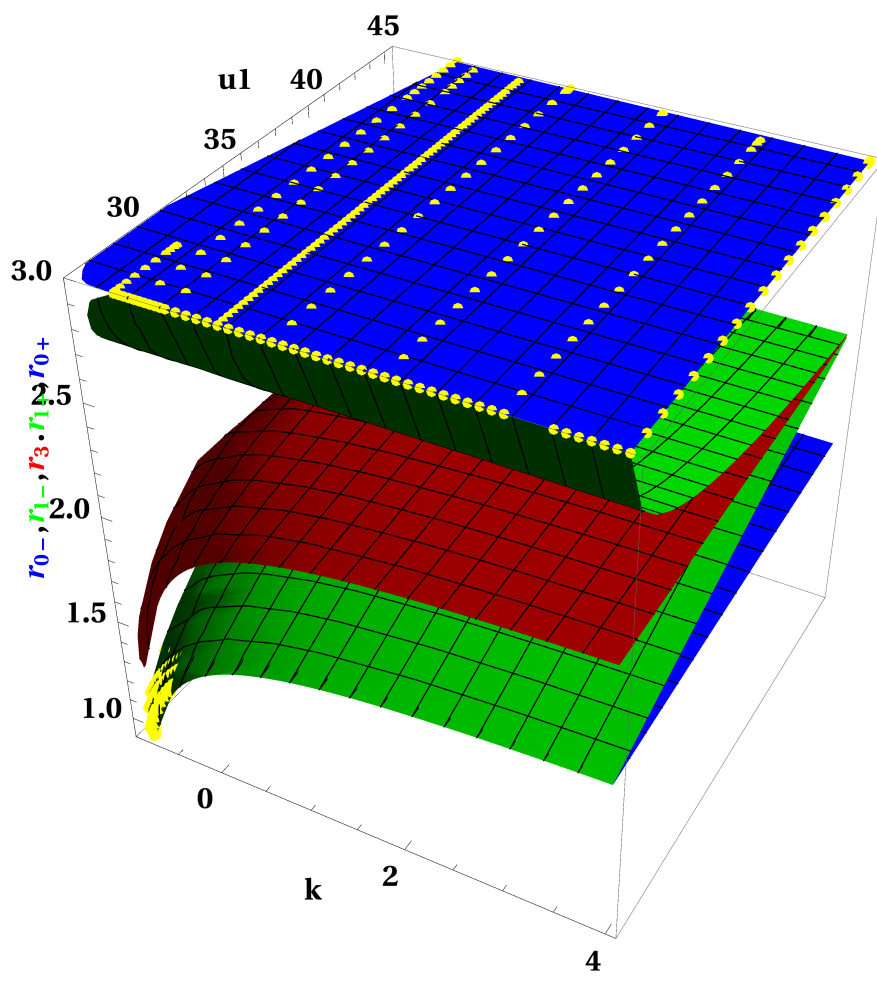

FIG. 4. From bottom to top, $r_{0-}, r_{1-}, r_{3}, r_{1+}$ and $r_{0+}=3$ against $k$ and $u_{1}$, for Ansatz 1. The colour coding is the same as in Figs. 1 and 3 The yellow beads indicate the discrete solutions we have computed, while surfaces represent interpolations. The plot ranges are $-1 \leq k \leq 4$ and $u_{0} \leq u_{1} \leq u_{*}$. The top of the box is at $r_{0}=3$.

$k$ or smaller $u_{1}$ give rise to less compact (smaller $\Gamma$ ), more centrally redshifted (smaller $\alpha_{c}$ ) and more spatially extended (smaller $r_{1-}$ and larger $r_{1+}$ ) solutions, sitting in a wider potential well (smaller $r_{0-}$ ). (To be accurate, $r_{1+}$ only depends on $u_{1}$.) By contrast, the most compact, least spatially extended and least centrally redshifted solution appears to be the unique thin-shell solution, which

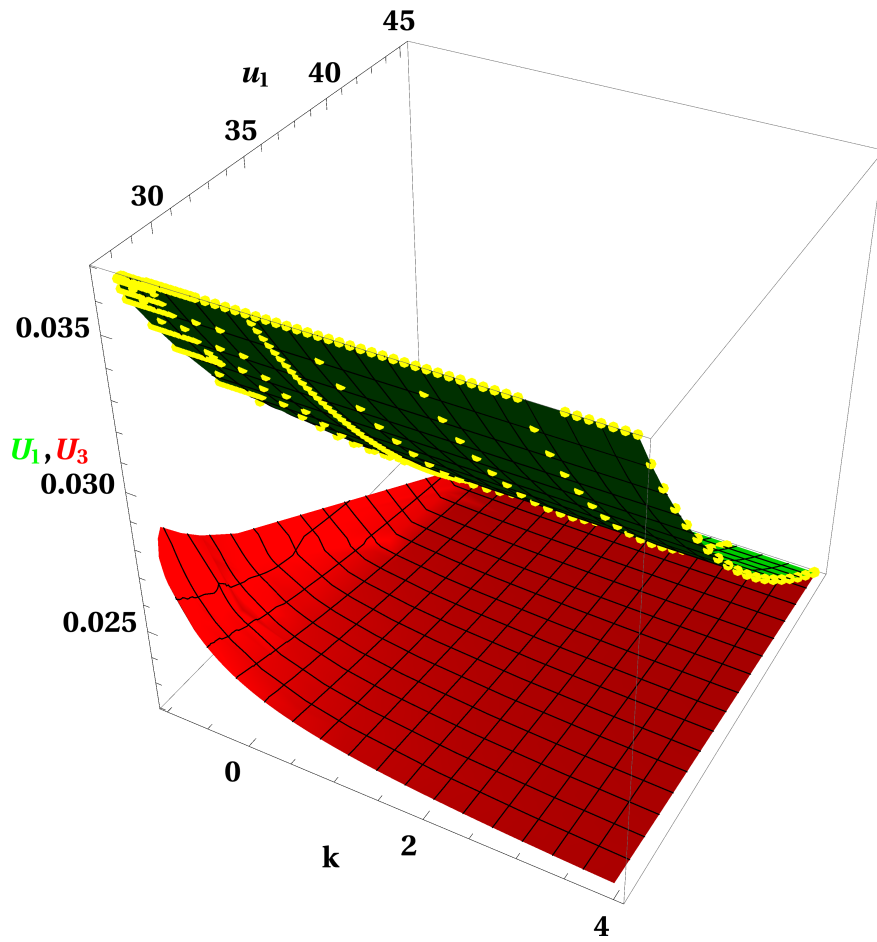

FIG. 5. $U_{3}$ (bottom) and $U_{1}$ (top) against $k$ and $u_{1}$, for Ansatz 1. The colour coding is the same as in Figs. 11 Fig. 3 and 4 . The top and bottom of the box are at $U_{0}=1 / 27$ and $U_{*}=729 / 16$, respectively. $U_{3}$ and $U_{1}$ come together at the value $U_{*}$ in the thin shell solution. In our family of solutions, this correspond to the limit $u_{1} \rightarrow u_{*}$, for any $k$, the back of the box.

is obtained in the limit $u_{1} \rightarrow u_{*}$, for any $k$.

Fig. 7 shows the projection into the $\left(Z_{c}, \Gamma\right)$-plane of the data points shown as yellow dots in Fig. 4. It is striking that these points lie in a narrow strip, raising the question if there is in fact a one-to-one relation between values of $Z_{c}$ and $\Gamma$, and the deviations from it seen in this figure are due to numerical error.

Our own numerical results cannot settle this question, as we have not attempted to quantify our numerical error. However, the equivalent Fig. 11 of [1], and in particular the inset of that figure, shows that their 1-parameter families $k=1,2,3,4$ (in the notation introduced in Appndix C) are distinct curves in the $\left(Z_{c}, \Gamma\right)$ plane. They actually plot 2-parameter families of ansätze $k(Q, F)$ that reduce to 1-parameter families $\bar{k}(Q)$. The fact that these 2-parameter families clearly appear as curves rather than areas in the $\left(Z_{c}, \Gamma\right)$ plane gives an indication that their numerical error is much smaller than the difference in the curves for different values of $k$.

We can also make a, non-rigorous, theoretical argument that $Z_{c}$ is not a function of $\Gamma$, using only Eq. 61. For the sake of argument, consider $\alpha_{0}$ as given, and $a_{0}$ obtained from it in closed form. $\Gamma$ depending one-to-one on $Z_{c}$ would then be equivalent to $\max _{r} a_{0}(r)$ depending only on $\alpha_{0}(0)$. Looking at the explicit expression for 


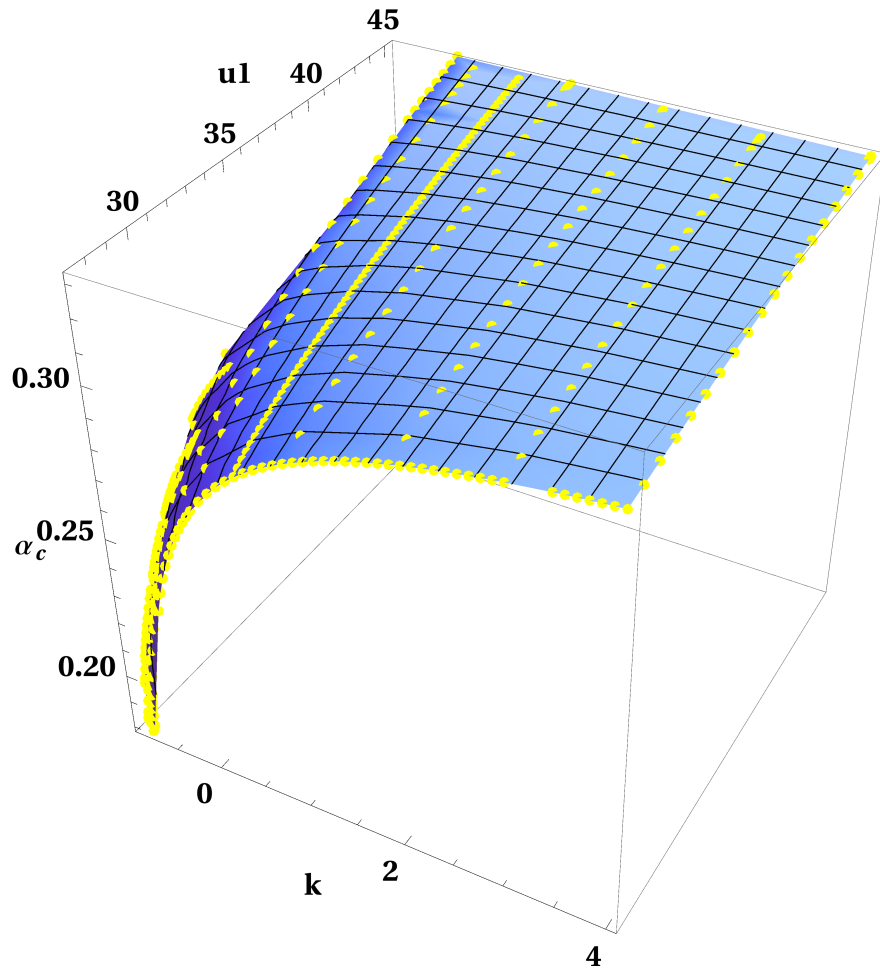

FIG. 6. $\alpha_{c}$ against $k$ and $u_{1}$, for Ansatz 1. The front of the box is at $u_{0}=27$, and the back at $u_{*}=729 / 16$. The top of the box is at $\alpha_{c *}=1 / 3$, the value for the thin shell solution. In our family of solutions, this corresponds to $u_{1} \rightarrow u_{*}$, for any $k$, the back of the box.

$a_{0}$, this seems implausible. Note that because this argument only uses Eq. (61), it holds for static spherically symmetric solutions with any tracefree effective matter, often called "geons" [10].

\section{THE SPACE OF STATIC SOLUTIONS}

Our mathematical results and numerical experiments suggest the following three conjectures concerning the space of solutions. The first two conjectures are an attempt at making more precise the notion that "locally" the space of static spherically symmetric solutions of the massless-Einstein Vlasov is a space of functions of one variable, subject to certain integrability and positivity conditions. The third conjecture is an attempt to characterise the subspace of solutions that are analytic in the matter region. For definiteness, we fix $M=1$.

1. For every function $\varphi(q)$ defined on the range $\left[u_{0}, u_{*}\right]:=[27,729 / 16]$, such that

(a) $\varphi$ is non-negative and not identically zero;

(b) for all $u$ in this range the integrals $\tilde{A}(u)$ and $\tilde{B}(u)$ defined in \begin{tabular}{r|r|}
77 & 78 \\
\hline
\end{tabular}

(c) $\tilde{A}(u)$ is finite;

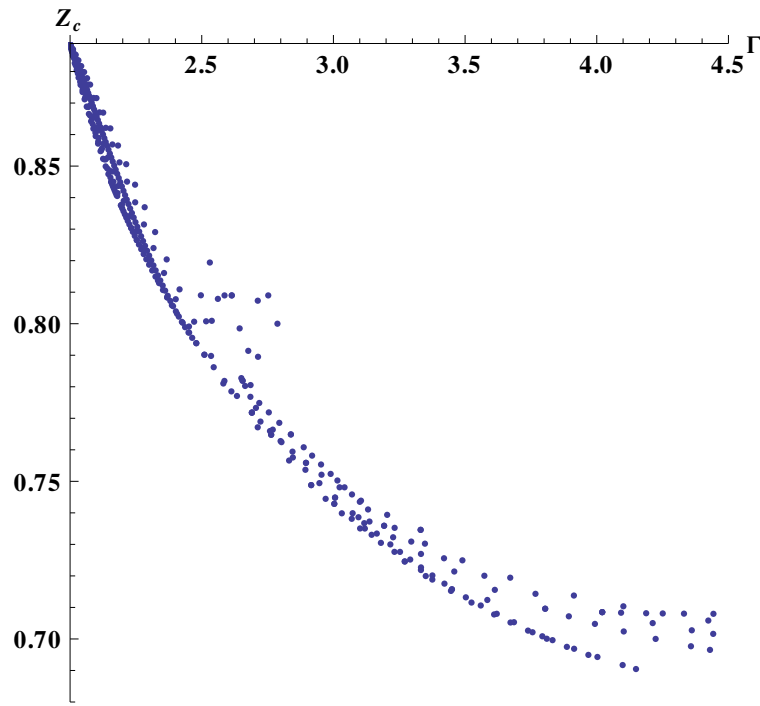

FIG. 7. Projection into the $\left(Z_{c}, \Gamma\right)$-plane of the solutions from Ansatz 1 shown as yellow beads in Figs 4 and 5 As discussed in the text, these dots fill a narrow region. However, as that narrow region is "almost" the graph of a monotonic function, and as we have already plotted $\alpha_{c}$ against $\left(k, u_{1}\right)$, there is no need for us to plot $\Gamma$ against $\left(k, u_{1}\right)$. In this plot we show $Z_{c}:=\alpha_{c}^{-1}-1$, rather than $\alpha_{c}$, for direct comparison with Fig. 11 of AC.

(d) $\tilde{B}(u)$ is either finite or it is singular at isolated points not including $u_{0}$ and $u_{*}$, such that $\tilde{B}(u)$ is integrable;

there exists a unique $C>0$ such that $C \varphi(q)$ creates a self-consistent solution of 8283 with $M=1$ and a single potential well.

2. Conversely, for every static spherically symmetric metric of the form 30 with $M=1$, such that

(a) $a_{0}$ and $\alpha_{0}$ are given by 65 with $0<\alpha_{c}<1$ for $0 \leq r<r_{1-}$, and by (66) with $M=1$ for $r>r_{1+}$, where $0<r_{1-}<r_{1+} \leq r_{0+}:=3$;

(b) for $r_{1-} \leq r \leq r_{1+} A(r)$ and $B(r)$ defined by (67 68 exist and are non-negative;

(c) $A(r)$ is finite;

(d) $B(r)$ is either finite or it is singular at isolated points not including $r_{0+}$ such that $B(r)$ is integrable;

(e) $a_{0}$ is $C^{1}$ and $\alpha_{0}$ is $C^{2}$ except at isolated points, and the two functions obey (61) for $r_{1-} \leq$ $r \leq r_{1}$ (in the sense of distributions at those isolated points);

(f) $u(r)$ defined by 74 is monotonically decreasing for $r_{1-}<r<r_{3}$ and monotonically increasing for $r_{3}<r<r_{1}$ for some $r_{3}$;

(g) $1 \leq a_{0}(r)<3$; 
there exists a consistent solution with this metric and a matter distribution $\varphi(q)$ given by 80 or (81).

3. In particular, a class of solutions where the metric is analytic in $r$ in the non-vacuum region $r_{1-} \leq r \leq$ $r_{1+}$ is obtained from the ansatz

$$
\varphi(q)=\sum_{n=0}^{\infty} C_{n}\left[\frac{q}{u_{1}}-1\right]_{+}^{n-\frac{1}{2}}
$$

provided the series converges uniformly in $u_{*} \leq q \leq$ $u_{1}$, and for either

(a) $u_{*}<u_{1}<u_{0}$; or

(b) $u_{1}=u_{0}$ and $C_{0}=0$,

If $C_{n_{0}}$ is the lowest non-vanishing coefficient in (103), the corresponding stress-energy tensor is given to leading order near the inner vacuum boundary by

$$
\begin{aligned}
& \rho(r) \sim\left[r-r_{1-}\right]_{+}^{n_{0}}, \\
& p(r) \sim\left[r-r_{1-}\right]_{+}^{n_{0}+1} .
\end{aligned}
$$

Similarly, in a one-sided neighbourhood of the outer vacuum boundary, the leading behaviour is

$$
\begin{aligned}
& \rho(r) \sim\left[r_{1+}-r\right]_{+}^{n_{0}}, \\
& p(r) \sim\left[r_{1+}-r\right]_{+}^{n_{0}+1},
\end{aligned}
$$

for $u_{1}>u_{0}$ (when $\left.u-u_{1} \sim r-r_{1+}\right)$ and

$$
\begin{aligned}
& \rho(r) \sim\left[r_{1+}-r\right]_{+}^{2 n_{0}}, \\
& p(r) \sim\left[r_{1+}-r\right]_{+}^{2\left(n_{0}+1\right)},
\end{aligned}
$$

for $u_{1}=u_{0}\left[\right.$ when $\left.u-u_{1} \sim\left(r-r_{1+}\right)^{2}\right]$.

We now make a number of comments, in the order of the conjectures.

1. For simplicity, we have excluded the thin-shell solution.

2. Conditions $1 \mathrm{a}$ and $2 \mathrm{a}$ are related, each expressing that we have a shell solution with a vacuum centre. The existence of a vacuum centre, that is $r_{1-}>0$, follows from finite central redshift, that is 88 with $\alpha_{c}>0$.

3. The integrability condition $1 \mathrm{~b}$ and the differentiability condition $2 \mathrm{~b}$ are related, expressing existence of the pressure and density.

4. Conditions $1 \mathrm{c}$ and $2 \mathrm{c}$ are related, as are $1 \mathrm{~d}$ and $2 \mathrm{~d}$, expressing positivity of the pressure and density, respectively.
5. Condition $1 \mathrm{~d}$ is a straightforward generalisation of the case where $\rho$ diverges at $u_{1}$ for $u_{1}>u_{0}$ to a case where $u_{1}$ is replaced by $u_{2}$, or by an isolated singularity inside the nonvacuum region (using the linearity of the relation between $\rho$ and $\varphi$ ). A similar comment applies to condition $2 \mathrm{~d}$.

6. In Conjecture 2, in a change of emphasis relative to Sec. IIIB, we have chosen to treat the metric as a single given entity obeying the trace-free constraint (61), rather than splitting the conjecture into two, one taking $a_{0}$ as given and $\alpha_{0}$ defined as the solution of (61), and vice versa for the other. It is possible that Conditions 2f] and 2g are redundant and can be derived from (61).

7. Condition 2f stipulates that there is a single potential well, and that it has a single minimum. As mentioned above, the extension to multishell solutions is straightforward because of Birkhoff's theorem.

8. Condition $2 \mathrm{~g}$ is given by the positivity of the mass, and the fact that no other solution can be as compact as the thin-shell solution [9], where $a_{0}\left(r_{*}\right)=3$ at the shell.

9. The ansatz 103 is a linear superposition of examples of our Ansatz 1. The system 8283 of two first-order ODEs is then quasilinear with analytic coefficients, which suggests that solutions that remain regular are in fact analytic. 104 109 follow from \begin{tabular}{ll|l|l|l|l|l|}
\hline 96 & 97 & 69 & 70 &
\end{tabular}

10. An obvious sufficient condition for the convergence of 103 is that there exist an $N>0, K>0$ and $R>u_{*}-u_{1}$ such that $0 \leq C_{n} \leq K R^{-n}$ for all $n>N$.

\section{THE CRITICAL SOLUTION}

AC have kindly given us tabulated data for $a_{0}(r)$ and $\alpha_{0}(r)$ for a representative of their approximately universal type-I critical solution obtained by fine-tuning generic initial data, corresponding to their Figs. 7 and 8. The solution resulting from the ansatz (95) with $u_{1}=u_{0}$ and $k=1 / 2$, corresponding to $\tilde{B}(u) \propto u-u_{0}$ and $\tilde{A}(u) \propto\left(u-u_{0}\right)^{2}$, is a good fit, to within the error of their numerical data (mostly a deviation from staticity). This is shown in Figs. 8 and 9.

In [5], we noticed the degeneracy of the massless case for both static and continuously self-similar solutions, and conjectured it for their linear perturbations, but we overlooked that it holds in general, as shown here in Sec. III. We went on to conclude that there could be no type-II critical phenomena because any critical solution would have an infinite number of growing modes $\delta f$ (but giving the same $\delta \bar{f}$ ) if it had one. As AC correctly noted, this argument, if correct, would equally hold for 


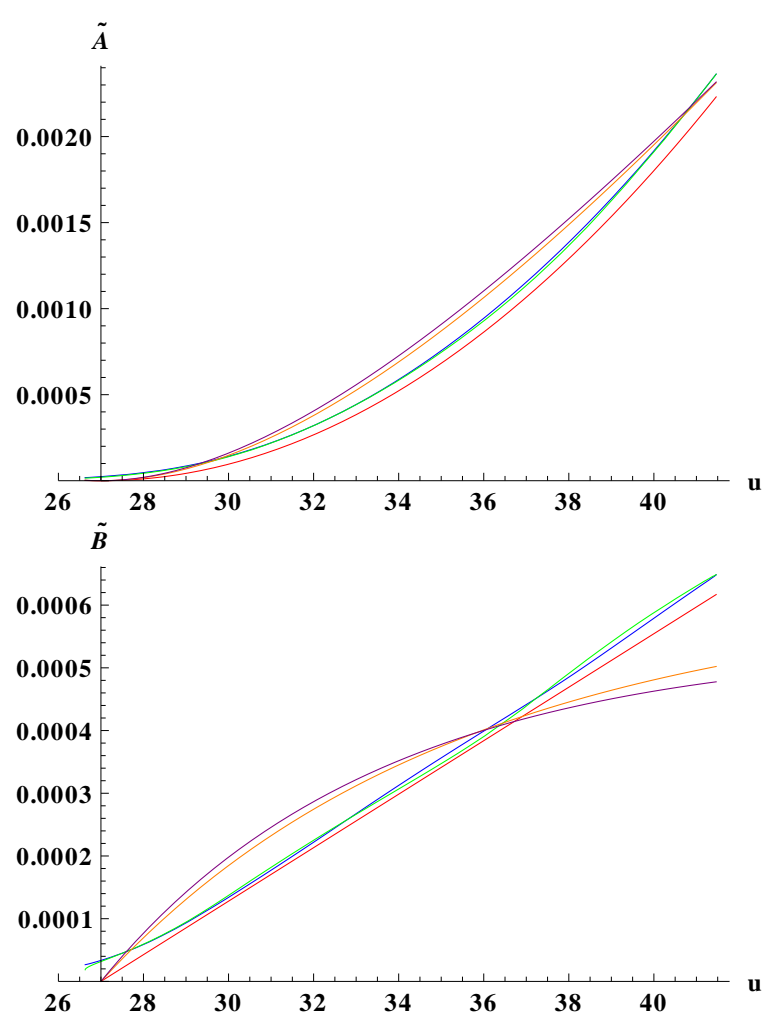

FIG. 8. Plot of $\tilde{A}(u)$ (upper plot) and $\tilde{B}(u)$ (lower plot) inferred from the numerical values of $a_{0}(r)$ and $\alpha_{0}(r)$ corresponding to Figs. 7 and 8 of 1, which represent the approximately universal critical solution. The three curves which lie almost on top of each other, correspond to the numerical data for $r_{0-}<r<r_{3}$ (blue) and $r_{3}<r<r_{0+}$ (green), and Ansatz 1 with $k=1 / 2$ and $u_{1}=u_{0}$ (red). The other two curves correspond to Ansatz 2 with $k=1 / 2$ and $u_{1}=u_{0}$ (orange) and Ansatz 3 with $k=0, l=-1 / 2$ and $u_{1}=u_{0}$ (purple). The axes origin is at $(27,0)$. Note that a selfconsistent solution cannot extend below $u=27$ while the numerical curves do, indicating that the data of [1] are not exactly static, either because of limited fine-tuning or because of numerical error.

type-I critical phenomena. However, there is no such argument within the reduced system. It remains entirely possible that the reduced system admits solutions that are 1-mode unstable (with a unique $\delta \bar{f}$ ).

In particular, if there exists a single 1-mode unstable static solution, this would explain Result (I) of AC, that at the black hole threshold there is a type-I critical solution with universal metric $\left(a_{0}, \alpha_{0}\right)$ up to an overall scale and universal critical exponent, but family-dependent $f$. (However, $\bar{f}$ would then also be universal up to scale.)

There is a tension between this and Result (II) of AC, that every static solution is at the threshold of collapse. Elsewhere in critical collapse, the critical solution is uniquely identified by being 1-mode unstable and at the threshold of collapse. If there is a continuum of solutions obeying these two criteria, what singles out the universal, or approximately universal, critical solution of

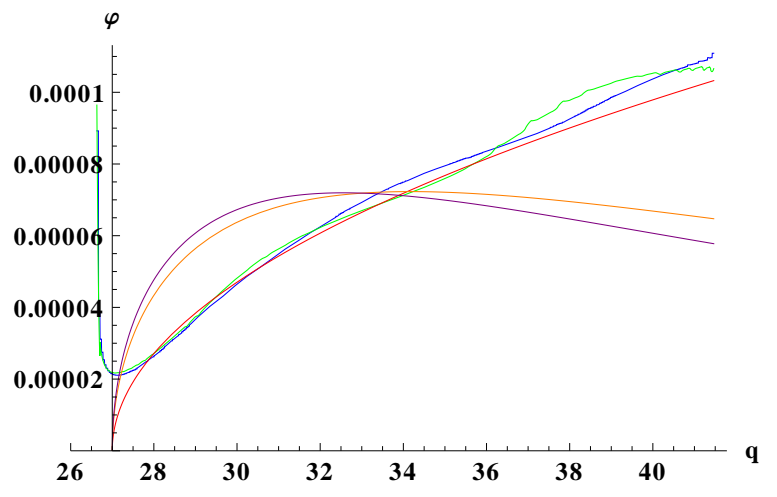

FIG. 9. Value of $\varphi(q)$ obtained from the critical metric of [1] via $\tilde{B}(u)$, using the formula 80 , and three exact solutions. The colour-coding of is the same as in Fig. 8. From this and the previous plot, it is clear that Ansatz 1 is a good approximation to the critical solution (at least for the families of initial data considered by AC), while Ansatz 2 and 3 are not. Again, the critical solution found in time evolutions extends below $u=27$, meaning that it cannot be exactly static.

AC's Result (I) among them?

We conjecture the following resolution of this apparent paradox.

1. Assume that in a near-critical time-evolution, a static critical solution is approached as the effective potential $U(t, r)$ becomes time-independent and any particles that are unbound in it evaporate to infinity. The potential well should then end up filled to the lip, or $U_{1}=U_{0}$ in our notation.

2. If the initial data are regular (for example smooth), we would expect the critical solution to be similarly regular, in particular at $U=U_{1}$. This selects a power-series solution of the form 103 with $u_{1}=u_{0}$ and $C_{0}=0$.

3. For initial data fine-tuned to the collapse threshold, but which are otherwise generic, all terms in (103) should be present, starting in particular from $C_{1} \neq$ 0 . However, we have no argument for setting the higher $C_{n}$ in (103). We conjecture that the critical solution is in fact only approximately universal.

4. There seems to be good agreement of $\varphi(q) \propto[q-$ $\left.27 M^{2}\right]_{+}^{1 / 2}\left(\right.$ Ansatz 1 with $k=1 / 2$ and $u_{1}=27 M^{2}$ ) with the observed critical solution, as we demonstrated in Figs. 8 and 9. By comparison, Ansatz 2 with $k=0, l=-1 / 2$ and $u_{1}=u_{0}$, and Ansatz 3 with $k+l+1=1 / 2$ and $u_{1}=u_{0}$, both of which have the same leading power of $q-u_{1}$, but differ in higher powers, do not fit at all (see again Figs. 8 and 9. We can only guess that families of initial data sufficiently different from the ones evolved by AC would show a significantly different critical solution. 


\section{CONCLUSIONS}

We have reformulated the Einstein-Vlasov system in spherical symmetry in a way in which the limit of vanishing particle mass $m$ is transparent. We have used this to show that the space of massless static solutions is essentially a space of functions of one variable. This function can be taken to be any one of $a_{0}(r), \alpha_{0}(r)$, or $\varphi(q)$. Moreover, given either one of the metric coefficients, we can give $\varphi(q)$ in closed form. Conversely, $\varphi(q)$ determines the two metric coefficients through an ODE boundary value problem. Modulo the conjecture that this has a unique solution, there is then a one-to-one correspondence between these different ways of characterising a static solutions. The detailed conjecture is given Sec. V.

It is clear from our numerical examples that static spherically symmetric solutions of Einstein-Vlasov with massive particles are highly general-relativistic. An interesting open question is therefore what solutions achieve the smallest inner radius of the matter shell, the lowest maximal compactness $\Gamma$ and largest central redshift $Z_{c}$. A priori this could be three different solutions, or families of solutions, but our numerical plots suggest that these are achieved by a single unique solution, given by the limit $k \rightarrow-1$ and $u_{1} \rightarrow u_{0}$. (However, the limiting point $k=-1, u_{1}=u_{0}$ is not itself part of the solution space).

By contrast, we already know that the most compact, least spatially extended and least centrally redshifted solution is the unique thin-shell solution, where the shell is at $r=9 M / 4$ and hence $\Gamma=8 / 9$ and $Z_{c}=2$. In any case, by considering $k$ down to -1 , we have already explicitly constructed solutions with compactness as low as $\Gamma \simeq 0.7$, whereas $A C$ only considered $k \geq 1$ and $[10$ seem to have explored only values of the equivalent parameter $k+l+1 \geq 0.7$.

We have also gone part of the way towards understanding the recent numerical results of Akbarian and Choptuik on the stability of static solutions and type-I critical collapse in the massless spherically symmetric EinsteinVlasov system. We have shown that there is, contrary to an incorrect argument implicit in [5], no reason why static solutions cannot be 1-mode unstable, and so no contradiction with their Result (II). In Sec. VI we have attempted to also reconcile their Results (I) and (II) with each other: we conjecture that there is no unique critical solution, but that the family-dependent critical solutions all look similar if the initial data are sufficiently generic, in the sense that all allowed powers of $q-u_{*}$ are present in $\varphi(q)$.

We hope that this non-universality can be confirmed by more accurate numerical simulations of critical collapse, using a wider variety of 1-parameter families of initial data. Our reformulation of the massless system in terms of the dependent variable $\bar{f}$ and independent variables $(t, r, z)$ should help with this in two ways: Reducing the number of independent variables of the problem (not counting time) from 3 to 2 allows a significant increase in resolution without loss of generality, while separating trivial from nontrivial parameters in the initial data allows fuller exploration of the space of generic initial data.

Clearly, in order to fully understand type-I critical phenomena, we need to obtain the perturbation spectrum of static solutions. In particular, we would like to identify the apparently unique unstable mode of all static solutions and understand its apparent universality.

We note for possible future use that in axisymmetry the angular momentum component $L_{z}$ is still conserved, and so we can adapt the formalism introduced here to reduce the axisymmetric massless Einstein-Vlasov system by one independent variable, from $f\left(t, \rho, z, p_{\rho}, p_{z}, L_{z}\right)$ to $\vec{f}\left(t, \rho, z, p_{\rho} / L_{z}, p_{z} / L_{z}\right)$, where, in this expression only, $\rho, z, \varphi$ are the standard cylindrical coordinates. This means that the number of independent variables (not counting time) reduces from 5 to 4 .

\section{ACKNOWLEDGMENTS}

I am grateful to Håkan Andréasson and Simone Calogero for inviting me to Göteborg, to Håkan, Maximilian Thaller and Ellery Ames for helpful and inspiring discussions that gave rise to this paper, and to Chalmers University of Technology for financial support. I am also grateful to Arman Akbarian for a discussion of his work and for making available numerical data relating to the critical solution, and to Matt Choptuik for comments on a draft.

\section{Appendix A: Dimensional analysis}

In gravitational units where $c=G=1$, all geometric quantities can be assumed to have dimensions that are powers of length $L$. In particular, we can assume that

$$
\left[T_{\mu}^{\nu}\right]=L^{-2}, \quad[a]=[\alpha]=1, \quad[t]=[r]=[M]=L,
$$

where the metric is given by (7). In Einstein-Vlasov, it is both consistent and convenient for dimensional analysis to formally retain a separate dimension $P$ of particle momentum. For massive particles, momentum is particle mass $m$ times velocity (which is dimensionless for $c=1$ ), so $m$ also has dimension $P$. We then have

$$
\begin{aligned}
& {\left[p_{r}\right]=[m]=[E]=P, \quad[F]=P^{2} L^{2},} \\
& {[f]=[k]=P^{-4} L^{-2}, \quad\left[d V_{p}\right]=P^{2} .}
\end{aligned}
$$

We have defined our new variables so that powers of $P$ cancel, namely

$$
\begin{aligned}
& {[z]=[Z]=L^{-1},} \\
& {[U]=[Q]=L^{-2}, \quad[\bar{f}]=[\bar{k}]=L^{2},} \\
& {[u]=[q]=L^{2}, \quad[\varphi]=L^{-2},} \\
& {[A]=L, \quad[B]=L^{-1} .}
\end{aligned}
$$

How particle momentum $P$ scales with $L$ is a matter of convention, and has no physical significance in the 
context of the massless Einstein-Vlasov system. However, if one insists on giving this physical significance, one natural choice is that particle momentum scales as spacetime momentum, and so $P=L$. This corresponds to increasing the mass and size of a static solution with massless particles by using the same number of particles but scaling up their momenta. Another natural choice is that linear particle momentum is invariant under rescaling (because in the massive it would be natural for $m$ to be invariant), and so $P=1$. This corresponds to scaling up the mass and size of a static solution with massless particles by using more particles of the same momentum.

\section{Appendix B: Expansion of the shooting equations about $u_{1}$}

With 9697, for $-1 \leq k<-1 / 2, u$ and $b$ are continous but $b^{\prime}$ diverges at $r=r_{1 \pm}$. Hence to start up a numerical solution of 8283 we need to expand the solution about these singular points. We make the ansatz

$$
\begin{aligned}
& u(r) \simeq u_{1}+\alpha\left(r-r_{1}\right)^{p}, \\
& b(r) \simeq b_{1}+\beta\left(r-r_{1}\right)^{q},
\end{aligned}
$$

and retain only the leading power of $r-r_{1}$ in each of the three terms in (8283). We can then attempt to match the power and coefficient in two of these terms in each equation, thus giving us four algebraic equations for $(\alpha, \beta, p, q)$, and then verify that neglecting the remaining term in each equation is self-consistent for the resulting values of $(p, q)$. Neglecting the third term in both (82) and 83 gives the linearised vacuum Einstein equations, and so is not a relevant approximation, leaving us with $3 \cdot 3-1=8$ other possible combinations of neglected terms. We need to distinguish three values of $b_{1}$, as follows.

Matching to the interior vacuum region In this special case $b\left(r_{1-}\right)=1$, and so we can approximate $1-3 b \simeq$ -2 in 82 . Neglecting the third term of 82 and the second term of (83), we find

$$
\begin{aligned}
u-u_{1} & \simeq 2 u_{1} \frac{r-r_{1-}}{r_{1-}}, \\
b-1 & \simeq-\hat{C}_{-} 2^{k+3 / 2}\left(\frac{r-r_{1-}}{r_{1-}}\right)^{k+3 / 2},
\end{aligned}
$$

where we have defined the shorthands

$$
\hat{C}_{ \pm}:=\frac{8 \pi^{2} u_{1}}{r_{1 \pm}^{2}} C
$$

The conditions for the two neglected terms to really be subdominant reduce to $k>-3 / 2$. Any other choice of neglected terms is inconsistent.

Matching to the exterior vacuum region when the potential is full In this special case we have $r_{1+}=3$ and $u_{1}=27$ (as always setting $M=1$ ) and $b(3)=1 / 3$. It is clear that for $k<-1 / 2$ the second term of (83), which is finite, can be neglected with respect to the third term, which diverges as $u \rightarrow u_{0}$. If we neglect the third term of (82), we find consistent powers, but the coefficients are complex. In the other two cases the powers are not consistent. This suggests that there is no continuous solution with $k<-1 / 2$ and $u_{1}=u_{0}$.

Matching to the exterior vacuum region when the potential is not completely filled In the generic case we have $b_{1} \neq 1,1 / 3$, and $r_{1+}$ and $b_{1}$ are given in terms of $u_{1}$ by (87) and (89). Neglecting the third term of (82) and the second term of 83 , we find

$$
\begin{aligned}
& u-u_{1} \simeq u_{1} \frac{\left(1-3 b_{1}\right)}{b_{1}} \frac{r_{1+}-r}{r_{1+}} \\
& b-b_{1} \simeq-2 \hat{C}_{+}\left(\frac{1-3 b_{1}}{b_{1}}\right)^{k+1 / 2}\left(\frac{r_{1+}-r}{r_{1+}}\right)^{k+3 / 2}
\end{aligned}
$$

This is consistent for $-3 / 2<k<-1 / 2$ and the other two possibilities are not.

\section{Appendix C: Ansatz 2: Akbarian and Choptuik}

To obtain solutions that are static by construction, AC make the ansatz

$$
h(E, F)=C_{\mathrm{AC}}\left[1-\frac{E}{E_{0}}\right]_{+}^{k} \delta\left(F-F_{0}\right) .
$$

In our formalism this corresponds to

$$
\begin{aligned}
\varphi(q) & =C 2^{k} u_{1}^{-1}\left(\frac{u_{1}}{q}\right)^{2}\left[1-\sqrt{\frac{u_{1}}{q}}\right]_{+}^{k} \\
& \simeq C(k+1) u_{1}^{-1}\left[\frac{q}{u_{1}}-1\right]_{+}^{k},
\end{aligned}
$$

with the parameters in $(\mathrm{C} 2$ related to those in $(\mathrm{C} 1)$ by

$$
\begin{aligned}
u_{1} & =\frac{F_{0}}{E_{0}^{2}}, \\
C & =2^{-k}(k+1)^{-1} E_{0}^{2} C_{\mathrm{AC}} .
\end{aligned}
$$

Note that $C_{\mathrm{AC}}$ has dimension. We have normalised $C$ so that (C2) agrees to leading order with (95) for the same $C$. The ansatz $\mathrm{C} 2$ can be integrated in closed form for $k>-1$ to give

$$
\begin{aligned}
\tilde{A}(u)= & \tilde{C} u_{1}^{1 / 2}\left[\frac{u}{u_{1}}-1\right]_{+}^{k+\frac{3}{2}}\left(\frac{u_{1}}{u}\right)^{k+1} \\
& { }_{2} F_{1}\left(\frac{k+1}{2}, \frac{k+2}{2}, \frac{5}{k+1}, 1-\frac{u_{1}}{u}\right) \\
\simeq & \tilde{C} u_{1}^{1 / 2}\left[\frac{u}{u_{1}}-1\right]_{+}^{k+\frac{3}{2}}, \\
\tilde{B}(u)= & 2 \tilde{A}^{\prime}(u) \simeq \tilde{C}(2 k+3) u_{1}^{-1 / 2}\left[\frac{u}{u_{1}}-1\right]_{+}^{k+\frac{1}{2}}
\end{aligned}
$$


with $\tilde{C}$ again defined by 98 . In the limit $k \rightarrow-1$ we again obtain the single-orbit case (99,101).

Akbarian and Choptuik noted that all their explicitly constructed static solutions with the two parameters $F_{0}$ and $E_{0}$, for fixed $k$, lie on a single curve in $\left(Z_{c}, \Gamma\right)$. This is expected because this 2-parameter family in terms of $k(Q, F)$ corresponds to a 1-parameter family in terms of $\bar{k}(Q)$.

\section{Appendix D: Ansatz 3: Andréasson, Fajman and Thaller}

Andréasson, Fajman and Thaller [10] have proved the existence of solutions for the ansatz

$$
h(E, F)=\left[E_{0}-E\right]_{+}^{k}\left[F-F_{0}\right]_{+}^{l}
$$

with $l \geq-1 / 2, k \geq 0, F_{0} \geq 0$ and $E_{0}>0$. In our formalism this corresponds to

$$
\begin{aligned}
\varphi(q) & =C_{\mathrm{AFT}} u_{1}^{-1}\left(\frac{u_{1}}{q}\right)^{2} I_{k l}\left(\sqrt{\frac{u_{1}}{q}}\right) \\
& \simeq C(k+l+2) u_{1}^{-1}\left[\frac{q}{u_{1}}-1\right]_{+}^{k+l+1},
\end{aligned}
$$

where we have defined the shorthands

$$
\begin{aligned}
& I_{k l}(p):=\theta(1-p) \int_{p}^{1}(z-p)^{k}\left(1-z^{2}\right)^{l} z^{-(k+2 l+5)} d z \\
& C_{\mathrm{AFT}}:=2 E_{0}^{k+2} F_{0}^{l+1},
\end{aligned}
$$

$u_{1}$ is again given by (C4), and $C$ is given by

$$
C=C_{\mathrm{AFT}} 2^{k+1} \frac{\Gamma(k+1) \Gamma(l+1)}{\Gamma(k+l+3)} .
$$

Note that

$$
\left(\frac{d}{d p}\right)^{k+1} I_{k l}(p)=k !\left(1-p^{2}\right)^{l} p^{-(2 l+5)} .
$$

We have normalised $C$ so that this ansatz has the same leading term as Ansatz 1 and 2, with the combination $k+l+1$ here corresponding to our single parameter $k$. Note that (D1) does not explicitly have an overall adjustable parameter, as the authors take the approach where $h(E, F)$ is fixed and the mass is found by shooting. Moreover, the implied parameter $C_{\mathrm{AFT}}$ has dimension.

Translating the parameters $E_{0}$ and $F_{0}$ into our notation $u_{1}$ and $C$ clarifies their role: solutions exist with $27<u_{1} / M^{2} \leq 729 / 16$ and $k+l+2 \leq-1$, or $u_{1} / M^{2}=27$ and $k+l+2 \leq-1 / 2$, and the shape of the solution (up to an overall scale) depends only on $u_{1} / M^{2}$ and the powers $k$ and $l$. This means that solutions exist precisely for $C_{\mathrm{AFT}}$ (interpreting this dimensional constant as dimensionless by an implicit choice of units for $E_{0}$ and $F_{0}$ ) in an interval that depends on $k$ and $l$, and within that interval the value of $C_{\mathrm{AFT}}$ determines $u_{1} / M^{2}$.
[1] A. Akbarian and M. W. Choptuik, Phys. Rev. D 90, 104023 (2014)

[2] C. Gundlach and J. M. Martín-García, Living Rev. Relativity, 2007-05 (2007).

[3] H. Andréasson and G. Rein, Class. Quant. Grav. 23, 3659 (2006).

[4] H. Andréasson and G. Rein, Class. Quant. Grav. 24, 1809 (2007).

[5] J. M. Martín-García and C. Gundlach, Phys. Rev. D 65, 084026 (2002)

[6] A. D. Rendall and J. J. L. Velazquez, Veiled singularities for the spherically symmetric massless Einstein-Vlasov system, arXiv:1604.06576, unpublished.
[7] Abel Integral Equation, Encyclopedia of Mathematics, Kluwer Academic Publishers 2002, accessed online at http://www.encyclopediaofmath.org/index. php/Abel_integral_equation on 19 August 2015.

[8] A. D. Polyanin and A. V. Manzhirov, Handbook of Integral Equations, Second Edition, Chapman and Hall/CRC Press, Boca Raton, 2008. Content accessed online at http://eqworld.ipmnet.ru/en/solutions/ie/ ie-toc1.htm on 14 August 2015.

[9] H. Andréasson, J. Diff. Eq. 245, 2243 (2008).

[10] H. Andréasson, D. Fajman and M. Thaller, Models for Self-Gravitating Photon Shells and Geons, arXiv:1511.01290, unpublished. 